$64^{\text {ème }}$ Congrès de la SFCO, 03003 (2016)

DOI: $10.1051 / \mathrm{sfco} / 20166403003$

(C) Owned by the authors, published by EDP Sciences, 2016

\title{
POSTER
}

\section{Fibrome amélobastique : une entité nosologique à ne pas confondre}

\author{
Campana $\mathrm{F}^{1}$, Catros $\mathrm{S}^{2}$, Lombardi $\mathrm{T}^{3}$ \\ 1 Centre Massilien de la Face. Marseille \\ 2 Pole d'odontologie et de santé buccale. CHU de Bordeaux
}

L'améloblastome est une tumeur bénigne des maxillaires. Lorsque du tissu ectomesenchymateux dentaire calcifié ou pas est associé, trois formes sont décrites : le fibrome améloblastique, le fibrodentinome améloblastique et le fibro-odontome améloblastique. Ces tumeurs se développent chez des sujets plus jeunes et ne possèdent pas le caractère agressif local de l'améloblastome. Nous rapportons un cas de fibrome-améloblastique chez un enfant de 8 ans en insistant sur l'importance de la confrontation anatomo-clinique.

\section{Observation}

Un garçon de 8 ans consultait pour la découverte fortuite sur l'orthopantomogramme de l'agénésie de la dent 45 associée à une image mixte sous la dent 85 bordée par un liseré d'ostéocondensation. Cet enfant ne présentait aucun antécédent médical. II n'existait pas de tuméfaction intra-buccale. Il était décidé de réaliser l'extraction de la 85 et l'exérèse de la lésion osseuse sous anesthésie générale. L'histologie était en faveur d'une tumeur améloblastique. Une deuxième lecture de lame était demandée afin de préciser le type d'améloblastome. Elle concluait à un fibrome améloblastique (FA). Une surveillance était instaurée. II n'existait pas de récidive à 9 mois. Le patient était perdu de vue.

\section{Discussion}

L'améloblastome est par fréquence la deuxième tumeur bénigne des maxillaires. II s'agit d'une tumeur épithéliale, sans composante ectomesenchymateuse dentaire. Lorsque la prolifération épithéliale est associée à du tissu ectomesenchymateux dentaire calcifié ou pas, trois formes sont décrites : le fibrome améloblastique, le fibrodentinome améloblastique et le fibro-odontome améloblastique. Ces variétés sont rares et représentent probablement divers stades de maturation de la lésion. À l'inverse des améloblastomes « conventionnels », les FA ont été rapportées chez l'enfant jeune. La revue de littérature de Buchner sur 172 cas de FA montre des âges allant de 1 mois à 57 ans avec une moyenne de 14,9 ans. La découverte se fait en général dans la première ou deuxième décade, sans prédilection de sexe ou de localisation. La découverte est souvent fortuite lors de la réalisation d'une radiographie panoramique. Parfois la lésion peut déformer les corticales ou être découverte lors d'un retard d'éruption. Radiologiquement, il s'agit d'une image radio claire uni ou multiloculaire, plus ou moins volumineuse, contenant dans les formes matures une part variable de tissu calcifié amélaire ou dentinaire. Macroscopiquement, la lésion se présente comme une masse tumorale encapsulée. Microscopiquement, on observe un stroma tantôt lâche, tantôt fibreux, par endroits riche en fibroblastes, dans lequel on retrouve 
des travées d'épithélium en double couche avec des cellules externes organisées en palissade et internes à type de réticulum étoilé et des îlots de structure analogue au bourgeon dentaire. Le diagnostic différentiel inclut les différents améloblastomes et le fibrosarcome améloblastique. L'agressivité locale décrite pour les améloblastomes n'est pas rapportée pour les FA et la récidive est rare. La prise en charge est donc différente de l'améloblastome, nécessitant une exérèse simple. Le suivi à long terme est recommandé.

Ce cas illustre l'importance de la confrontation anatomo-clinique dans les tumeurs à composantes épithéliales de type améloblastome, en particulier chez l'enfant. La prise en charge thérapeutique, le pronostic et le suivi sont différents.

\section{Références}

Barnes $L$ et al. Pathology and genetics of head and neck tumours. Lyon: IARC Press; 2005.

1. Buchner A, Vered M. Ameloblastic fibroma : a stage in the development of a hamartomatous odontoma or a true neoplasm ? Critical analysis of 162 previously reported cases plus 10 new cases. Oral Surg Oral Med Oral Pathol Oral Radiol 2013;116: 598-606.

2. De Riu $\mathrm{G}$ et al. Ameloblastic fibro-odontoma. Case report and review of the literature. Journal of Cranio-Maxillo-Facial Surgery $2010 ; 38: 141 \mathrm{e} 144$. 